\title{
Cortisol-induced increases of plasma oxytocin levels predict decreased immediate free recall of unpleasant words
}

\section{Mattie Tops ${ }^{1,2,3}$ *, Femke T. A. Buisman-Pijlman ${ }^{4}$, Maarten A. S. Boksem ${ }^{5,6}$, Albertus A. Wijers $^{2}$ and Jakob Korf ${ }^{7}$}

${ }^{1}$ Centre for Child and Family Studies, University of Leiden, Leiden, Netherlands

2 Department of Experimental Psychology, University of Groningen, Groningen, Netherlands

${ }^{3}$ Leiden Institute for Brain and Cognition, Leiden University Medical Center, Leiden, Netherlands

${ }^{4}$ Discipline of Pharmacology, Faculty of Health Sciences, University of Adelaide, Adelaide, SA, Australia

${ }^{5}$ Donders Institute for Brain, Cognition and Behaviour, Radboud University, Nijmegen, Netherlands

${ }^{6}$ Rotterdam School of Management, Erasmus University, Rotterdam, Netherlands

${ }^{7}$ Department of Psychiatry, University Medical Center Groningen, Groningen, Netherlands

\section{Edited by:}

Eero Vasar, University of Tartu, Estonia

\section{Reviewed by:}

Sara Morley-Fletcher,

CNRS-University Lille, France

Sulev Kõks, University of Tartu,

Estonia

\section{*Correspondence:}

Mattie Tops, University of Leiden,

Centre for Child and Family Studies,

Post box 9555, 2300 RB Leiden,

Netherlands.

e-mail: topsm@fsw.leidenuniv.nl
Cortisol and oxytocin have been shown to interact in both the regulation of stress responses and in memory function. In the present study we administered cortisol to 35 healthy female subjects in a within-subject double-blind placebo-controlled design, while measuring oxytocin levels, adrenocorticotropic hormone (ACTH) levels, and free recall of pleasant and of unpleasant words. We found that cortisol administration suppressed ACTH levels and (1) induced a decrease in oxytocin associated with ACTH suppression and (2) an increase in oxytocin that was independent from ACTH suppression. This cortisol-induced increase in plasma oxytocin was associated with a selective decrease in immediate free recall of unpleasant words from primacy positions. The present results add to evidence that cortisolinduced increases in oxytocin could mediate some of the effects of stress and cortisol on memory, and possibly play a role in the regulation of the hypothalamo-pituitary-adrenal stress response. This mechanism could significantly impact affective and social behaviors, in particular during times of stress.

Keywords: oxytocin, cortisol, adrenocorticotropic hormone, memory, stress, hypothalamo-pituitary-adrenal axis, vasopressin

\section{INTRODUCTION}

As part of the hypothalamo-pituitary-adrenal (HPA) axis, corticotrophin releasing factor (CRF) is released by the hypothalamus to trigger release of adrenocorticotropic hormone (ACTH) and vasopressin into the bloodstream at the pituitary, which trigger release of glucocorticoids including cortisol from the adrenal cortex. Oxytocin is secreted from the same hypothalamic nuclei which regulate plasma vasopressin and the HPA axis, and neuroanatomic/electrophysiologic data indicate it is modulated by the same hippocampal projections which modulate plasma vasopressin and HPA axis activity (Risold and Swanson, 1996; Herman et al., 2002). Plasma oxytocin levels have been shown to be correlated positively with the integrity of hippocampal-mediated HPA feedback (Goldman et al., 2008). Additionally, in patients with Addison's disease, elevated ACTH levels were accompanied by higher oxytocin levels (Grenbäck et al., 2007). It has been suggested that cortisol-induced increases in oxytocin may be involved in the feedback regulation of the HPA axis (Liberzon and Young, 1997; Carter, 1998).

Based on findings suggesting that cortisol administration increases plasma oxytocin levels (Kalin et al., 1985; Tops et al., 2007b), we previously postulated the hypothesis that cortisol administration may have beneficial effects on stress coping and emotional memory that are partially mediated by increases in oxytocin (Tops et al., 2007a,b): oxytocin inhibits or downregulates stress responses, seems to inhibit acquisition and/or consolidation of aversive, high arousal experiences, and has analgesic effects (Uvnäs-Moberg, 1998; Lee et al., 2009).

However, these cortisol-oxytocin interactions in stress and memory function appear to be highly context dependent. Heinrichs et al. (2004) found that intranasal oxytocin administration impaired recall depending on the kind of memory test used and the psychobiological relevance of the stimuli, impairing recall of reproduction-related words but not neutral words in an implicit conceptual task. Intranasal has been found to increase the familiarity of previously presented faces (Rimmele et al., 2009). Animal research has shown that oxytocin is positively involved in social memory (Young, 2002), while high levels of oxytocin seem to inhibit acquisition and/or consolidation of aversive, high arousal experiences such as during labor (e.g., Silber et al., 1990). So it seems that effects of oxytocin on memory may, like effects of cortisol (De Kloet et al., 1999; Lupien et al., 2005), depend on arousal, and context. In the present study we investigated whether administration of cortisol to healthy female subjects increases plasma oxytocin levels, and whether increased oxytocin levels are associated with decreased immediate free recall of unpleasant but not 
of pleasant words. We measured plasma ACTH levels to include as predictor in the analyses, as cortisol administration suppresses plasma ACTH levels (Tops et al., 2006, 2007c) and may affect memory and plasma oxytocin levels through this route as well (Grenbäck et al., 2007). For the same purpose we will measure vasopressin, which is also a cortisol releasing factor. Our first aim is to replicate the finding that cortisol administration increases plasma oxytocin levels. Our second aim is to investigate whether the cortisol-induced increase in oxytocin level is associated with decreased immediate free recall of unpleasant words.

\section{MATERIALS AND METHODS PARTICIPANTS}

Thirty-five healthy female paid volunteers aged 30-57 $(M=45$, $\mathrm{SD}=8$ ) were enlisted by advertisement in a local newspaper. Each participant passed a health screening. Inclusion criteria included Dutch as their native language, regularly cycling, or using oral contraception, normal or corrected to normal vision, and no personal history of psychiatric, metabolic, or neurological disorders, or substance abuse. Volunteers who reported noxious health behaviors (drug abuse including excessive alcohol, smoking and caffeine, and abnormal sleeping habits, e.g., too little sleep), chronic health problems or psychopathology were excluded from the study. Our subjects self-reported the phase of their menstrual cycle based on days since onset of their last menstrual period, and active contraceptive use. All participants read and signed an informed consent statement approved by the Medical Ethical Committee of the University Medical Center of Groningen.

\section{PROCEDURE}

The subjects took part in a within-subjects double-blind design in which they were treated with either cortisol or placebo in two separate sessions, the order of which was counterbalanced and were separated by approximately 1 week. The participants arrived between 9:00 and 10:30. First, a capsule was ingested containing either cortisol (35 mg of hydrocortisone) or a placebo (avicel capsule). Participants were then allowed to read while they waited $70 \mathrm{~min}$ for a blood sampling. After that, the subjects performed the memory task which took $20 \mathrm{~min}$ to complete.

\section{MEMORY TASK \\ Word lists}

The to-be memorized pleasant and unpleasant nouns consisted of one to three syllables and were selected from two different databases. These words were rated in a pilot study for the degree of pleasantness of each word. Only words with relatively high emotional impact were used. The memory task consisted of two lists of pleasant words (e.g., "friendship") and two lists of unpleasant words (e.g., "hate"). Each word list contained 11 words from only one valence category. We constructed two parallel versions of the memory task, of which the order of presentation was counterbalanced across sessions. All lists were matched for frequency of written use, imageability, and word length. The pleasant words were matched with the unpleasant words for emotional impact. Examples of pleasant and unpleasant words translate to English as love, fun, loyalty, regard and resentment, cancer, violence, malaise, respectively. The lists of words can be obtained from the first author.

\section{Task}

The to-be memorized words were presented on a $15^{\prime \prime}$ computer screen for $2 \mathrm{~s}$ and were immediately replaced by the next word. Words were selected from one list at a time and were presented in random order. After each list, the participants were given $90 \mathrm{~s}$ to write down as many words as they could remember from the list, in any order (Tops et al., 2003, 2004; Rijsdijk et al., 2009), before moving on to the next list. The order of the lists from which the words were selected was also randomized.

\section{PLASMA HORMONE LEVELS}

The blood samples were collected in ice-chilled tubes. After centrifugation, the plasma was removed, and the samples were stored at $-80^{\circ} \mathrm{C}$ until analysis. Plasma concentrations of cortisol and ACTH were determined using an in-house radio immunoassay (Pratt, 1978). Vasopressin was extracted from the plasma using Sep-pak C 18 cartridges. The extracts were analyzed with a radioimmunoassay (MP-products, Amersfoort). These analyses were performed at the University Medical Center of Groningen. Before oxytocin was analyzed, plasma was extracted with acetone [Guarantee Reagent (GR); Merck, Darmstadt, Germany] and petroleum benzene (GR; boiling point range $40-60^{\circ} \mathrm{C}$; Merck) with a recovery of $92 \%$. Oxytocin levels were measured by radioimmunoassay at the Swedish University of Agricultural Sciences in Uppsala, Sweden, at the Department of Animal Physiology. The antibody $\mathrm{KA}_{19}$ was used for the analysis (Euro Diagnostica, Milab, Malmö, Sweden). The limit of detection was $4.68 \mathrm{pmol} / \mathrm{l}$ and interassay coefficients of variation low $22.06 \% \mathrm{CV}=9.57$; medium $37.83 \% \mathrm{CV}=8.81$; high $529.8 \% \mathrm{CV}=6.81$; intrassay $\% \mathrm{CV}<10$ conc $=20.90-1026$ (Stock and Uvnäs-Moberg, 1985, 1988).

\section{STATISTICAL ANALYSES}

The effects of cortisol administration on cortisol, ACTH (i.e., ACTH suppression) and vasopressin (vasopressin suppression) levels were analyzed using a General Linear Model (GLM) analysis with Condition (placebo vs. cortisol) as within-subject factor. To investigate the effects of cortisol administration on oxytocin levels, taking into account possible effects through ACTH suppression (level in the cortisol condition minus level in the placebo condition), a GLM analysis of oxytocin levels was performed with Condition (placebo vs. cortisol) as within-subject factor and ACTH suppression as continuous predictor.

Finally, to investigate whether cortisol-induced changes in oxytocin level are associated with changes in recall performance, we performed a GLM analysis of recall with Condition and Valence (pleasant vs. unpleasant words) as within-subject factors and with the effect of cortisol administration on oxytocin level (level in the cortisol condition minus level in the placebo condition) as continuous predictor. Means (SD) of hormone levels and recall are presented, while effects of continuous predictors are presented as Pearson's correlation coefficients.

\section{RESULTS}

We tried potential confounders, e.g., age, menstrual cycle phase, oral contraceptive use, and the order of treatments, as covariates. No potential confounders noteworthy altered the results, and they were not included in the final analyses. Cortisol and oxytocin levels 
were natural-log transformed to better approximate a normal distribution. There were two missing values for ACTH levels, reducing the number of subjects for analyses involving ACTH to 33.

\section{CORTISOL, ACTH, AND VASOPRESSIN}

A GLM analysis of cortisol levels with Condition (placebo vs. cortisol) as within-subject factor showed a main effect of Condition $[F(1,34)=181.16, p<0.000001]$. Cortisol levels were higher in the cortisol condition compared to the placebo condition (Table 1). A GLM analysis of ACTH levels with Condition (placebo vs. cortisol) as within-subject factor showed a main effect of Condition $(F(1,31)=34.41, p<0.00001)$. ACTH levels decreased in the cortisol treatment condition compared to the placebo condition (Table 1), reflecting a negative feedback mechanism. In contrast, there was no significant suppression effect of cortisol administration on vasopressin $(F(1,34)=2.41, p=0.130)$ although levels were somewhat lower in the cortisol condition compared to the placebo condition (Table 1).

\section{OXYTOCIN}

A GLM analysis of oxytocin levels with the effect of cortisol administration on ACTH level ("ACTH suppression"; level in the cortisol condition minus level in the placebo condition) as predictor showed that plasma oxytocin levels increased in the cortisol treatment condition compared to the placebo condition (Table 1; $F(1,31)=12.28, p<0.001)$. Additionally, there was an interaction between Condition and ACTH suppression $(r=0.58$, $F(1,31)=15.48, p<0.001)$ : subjects who showed strong suppression of ACTH levels actually displayed a decrease in oxytocin levels (see Figure 1A). A similar analyses with the effect of cortisol administration on vasopressin level as predictor yielded no significant results.

\section{MEMORY PERFORMANCE}

The mean proportion of words recalled was.64 $(\mathrm{SD}=0.31)$. As we previously showed differential effects of cortisol administration on primacy (the first four words in each list) compared to recency (the last four words) recall (Tops et al., 2004), we analyzed primacy recall and recency recall separately. A GLM analysis of primacy recall with Condition and Valence (pleasant vs. unpleasant words) as within-subject factors and with the effect of cortisol administration on oxytocin level as predictor showed an interaction between Condition, Valence, and oxytocin level $(F(1,33)=8.84, p=0.005)$. Following up this interaction by separate analyses of pleasant and unpleasant word primacy recall showed no effects for pleasant words, but unpleasant words

Table 1 | Mean plasma hormone levels (SD) per condition (placebo vs. cortisol treatment).

\begin{tabular}{lllll}
\hline & $\begin{array}{l}\text { Placebo } \\
\text { condition }\end{array}$ & SD & $\begin{array}{l}\text { Cortisol } \\
\text { condition }\end{array}$ & SD \\
\hline Cortisol (nmol/l) & 255 & 118 & 1213 & 456 \\
ACTH (ng/l) & 18.21 & 9.29 & 12.43 & 6.51 \\
Vasopressin (ng/l) & 0.89 & 0.66 & 0.81 & 0.53 \\
Oxytocin (nmol/l) & 52 & 12 & 55 & 14
\end{tabular}

recall displayed an interaction between Condition and oxytocin level $[F(1,33)=8.44, p=0.007]$. This cortisol-induced change in recall of unpleasant words was proportional to the change in oxytocin level: recall increased when oxytocin decreased and recall decreased when oxytocin increased $(r=-0.45, p=0.007)$. There was no effect on recall of pleasant words, as shown by the similar correlation between the change in oxytocin and the change in recall negativity bias (recall of unpleasant words minus recall of pleasant words; $r=-0.46, p=0.005$; see Figure 1B). There were also no significant results regarding recency recall. When included in the analyses, ACTH, and vasopressin did not significantly predict recall performance.

\section{DISCUSSION}

We showed that administration of cortisol increased plasma oxytocin levels, although subjects who showed strong suppression of ACTH levels actually displayed a decrease in oxytocin levels:

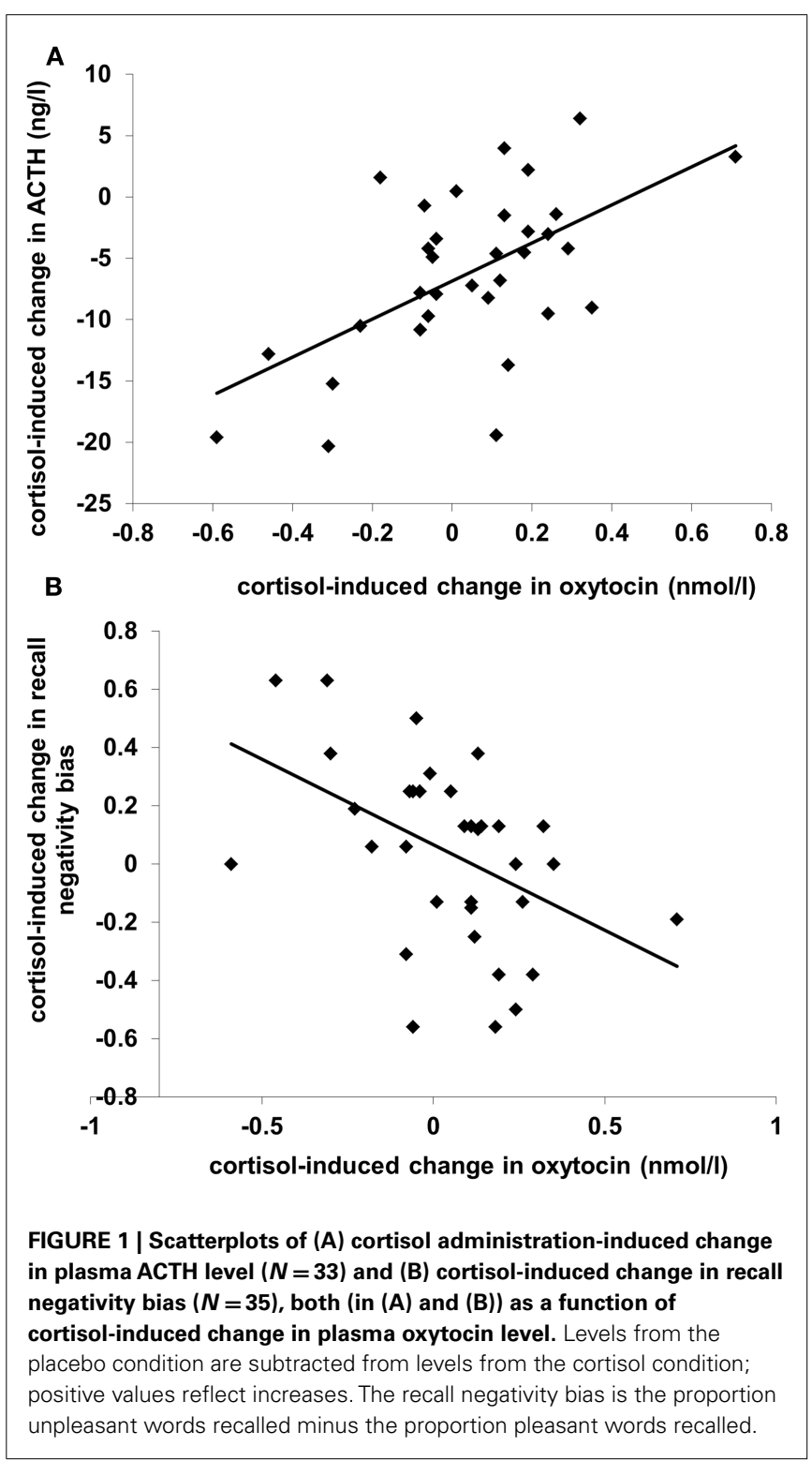


cortisol appeared to induce both a decrease in oxytocin associated with ACTH suppression and an increase in oxytocin that was independent from ACTH suppression. The observed cortisolinduced increase in plasma oxytocin was associated with a decrease in immediate free recall of unpleasant words from primacy (first) positions, but did not relate to recall of pleasant words or of words from recency (last) positions in lists of words.

Both CRF and oxytocin are secreted from the same hypothalamic nuclei that regulate the HPA axis, and are modulated by the same hippocampal projections which modulate HPA axis activity (Risold and Swanson, 1996; Herman et al., 2002). Plasma oxytocin levels have been shown to be correlated positively with the integrity of the hippocampal-mediated HPA feedback mechanism (Goldman et al., 2008). Indeed, it has been proposed that cortisol-induced increases in oxytocin may be involved in the feedback regulation of the HPA axis (Liberzon and Young, 1997; Carter, 1998) and that there may be important interactions between cortisol-mediated stress and oxytocin-mediated attachment. Stressful experiences (such as pregnancy and parturition), anxiety, neophobia, isolation, and separation often precede the formation of social attachments. Correlations between glucocorticoid levels and attachment offer support for a role of glucocorticoids in the events which eventually lead to pair bonding (Carter, 1998; Henry and Wang, 1998). Lower plasma oxytocin levels during pregnancy have been found to predict postpartum depression and risk for developing postpartum depression, suggesting that increased oxytocin during pregnancy could prevent postpartum depression (Skrundz et al., 2011). In addition, both positive social interactions and social bonds could function to provide a sense of safety and reduce anxiety or stress. In contrast, negative or traumatic social interactions and rejection could feed back to decrease oxytocin levels, heighten fear or anxiety and prevent the formation of social interactions (Carter, 1998).

Results from Dabrowska et al. (2011) suggest that CRF type 2 receptors located on oxytocinergic neurons and axon terminals might regulate the release of oxytocin and hence might be a crucial part of a potential feedback loop between the hypothalamic oxytocin system and the forebrain CRF system that could significantly impact on affective and social behaviors, in particular during times of stress. Other studies suggest that oxytocin release in response to stress may be modulated in part by high circulating glucocorticoids and their negative feedback on the release of hypothalamic CRF (Chen and Du, 1999). However, the positive association in the present study between cortisolinduced change in ACTH levels and change in oxytocin levels suggests that the negative feedback on hypothalamic CRF that is mediated by cortisol did not increase oxytocin levels. Nevertheless, cortisol-mediated feedback on CRF may be involved in both the decrease in oxytocin associated with ACTH suppression and the increase in oxytocin that was independent from ACTH suppression.

Recall from primacy positions, but not recall from recency positions, has been shown to depend on the integrity of the hippocampal formation (Kesner, 1985; Zola-Morgan and Squire, 1990). Although the exact mechanism cannot be inferred from this study, the results suggest the possibility that cortisol administration impacts on oxytocin levels and memory through effects on the hippocampus, or that cortisol administration increases oxytocin levels which impact on memory performance. Moreover, negative feedback by cortisol on the HPA axis may be involved.

In the present study we failed to find any direct effects of cortisol administration on immediate recall. A review of the effects of cortisol on memory in humans concluded that fairly consistently over studies, stress, or cortisol treatment does not affect immediate recall but temporarily blocks retrieval of long-term memory, especially of emotional arousing material, most likely through reduction of activity of the hippocampus (Wolf, 2009). In addition, cortisol appears to only influence memory when arousal is relatively high (e.g., due to venipuncture such as in the present study; Tops et al., 2006). In contrast to the effects on retrieval, cortisol enhances memory consolidation (Wolf, 2009). Because in the present study cortisol was administered before learning, decreased immediate recall most likely reflects effects on acquisition or retrieval. Future studies will have to determine whether association between cortisol-induced oxytocin-changes and reduced immediate recall of unpleasant words involves direct effects of oxytocin on retrieval, and whether such effects of oxytocin on retrieval are also involved in the effects of cortisol on retrieval from long-term memory. Moreover, future studies should employ receptor blockade to demonstrate direct involvement of oxytocin in mediating cortisol effects on memory.

Several aspects define this study as preliminary, such as reliance on single samples for hormone determination per session, inclusion of women only, and inclusion of subjects in various phases of their menstrual cycle. Nevertheless, the correlation between the oxytocin levels of the placebo and cortisol treatment conditions despite cortisol administration $(r=0.45, p=0.006)$ suggests reliability of oxytocin measurements. Consistent with larger studies employing between-subjects comparisons, we did not observe any relationships between menstrual cycle phase or oral contraceptive use and the present findings (Light et al., 2005). In contrast, studies employing a within-subject design report lower oxytocin levels in the luteal phase (Salonia et al., 2005), which suggests that menstrual cycle phase variations in oxytocin levels are small and might be easily masked by individual differences (Light et al., 2005).

The present results add to evidence that cortisol-induced increases in oxytocin could mediate some of the effects of stress and cortisol on memory, and may play a role in the regulation of HPA stress response. Cortisol-induced increases in oxytocin may facilitate stress coping and perhaps, by decreasing negative memory, decrease depressive responses to stress. This mechanism could significantly impact affective and social behaviors, in particular during times of stress.

\section{ACKNOWLEDGMENTS}

For reprints contact Mattie Tops, Centre for Child and Family Studies, University of Leiden, Post box 9555, 2300 RB Leiden, the Netherlands topsm@fsw.leidenuniv.nl. Mattie Tops was supported by an award from the Netherlands Organization for Scientific Research (Veni, NWO; www.nwo.nl). The funders had no role in study design, data collection and analysis, decision to publish, or preparation of the manuscript. 


\section{REFERENCES}

Carter, C. S. (1998). Neuroendocrine perspectives on social attachment and love. Psychoneuroendocrinology 23, 779-818.

Chen, X. Q., and Du, J. Z. (1999). Hypoxia induces oxytocin release in the rat. Neuro Endocrinol. Lett. 20, 373-378.

Dabrowska, J., Hazra, R., Ahern, T. H., Guo, J. D., McDonald, A. J., Mascagni, F., Muller, J. F., Young, L. J., and Rainnie, D. G. (2011). Neuroanatomical evidence for reciprocal regulation of the corticotrophinreleasing factor and oxytocin systems in the hypothalamus and the bed nucleus of the stria terminalis of the rat: Implications for balancing stress and affect. Psychoneuroendocrinology 36, 1312-1326.

De Kloet, E. R., Oitzl, M. S., and Joëls, M. (1999). Stress and cognition: Are corticosteroids good or bad guys? Trends Neurosci. 22, 422-426.

Goldman, M., Marlow-O'Connor, M., Torres, I., and Carter, C. S. (2008). Diminished plasma oxytocin in schizophrenic patients with neuroendocrine dysfunction and emotional deficits. Schizophr. Res. 98, 247-255.

Grenbäck, E., Hulting, A. L., Bucht, E., and Petersson, M. (2007). Plasma galanin, vasopressin, and oxytocin in patients with Addison's disease. Horm. Metab. Res. 39, 589-595.

Heinrichs, M., Meinlschmidt, G., Wippich, W., Ehlert, U., and Hellhammer, D. H. (2004). Selective amnesic effects of oxytocin on human memory. Physiol. Behav. 83, 31-38.

Henry, J. P., and Wang, S. (1998). Effects of early stress on adult affiliative behavior. Psychoneuroendocrinology 23, 863-875.

Herman, J. P., Tasker, J. G., Ziegler, D. R., and Cullinan, W. E. (2002). Local circuit regulation of paraventricular nucleus stress integration glutamate-GABA connections. Pharmacol. Biochem. Behav. 71, 457-468.

Kalin, N. H., Gibbs, D. M., Barksdale, C. M., Shelton, S. E., and Carnes, M.
(1985). Behavioral stress decreases plasma oxytocin concentrations in primates. Life Sci. 36, 1275-1280.

Kesner, R. P. (1985). Correspondence between humans and animals in coding of temporal attributes: role of hippocampus and prefrontal cortex. Ann. N.Y. Acad. Sci. 444, 122-136.

Lee, H. J., Macbeth, A. H., Pagani, J. H., and Young, W. S. III. (2009). Oxytocin: the great facilitator of life. Prog. Neurobiol. 88, 127-151.

Liberzon, I., and Young, E. A. (1997). Effects of stress and glucocorticoids on CNS oxytocin receptor binding. Psychoneuroendocrinology 22, 411-422.

Light, K. C., Grewen, K. M., and Amico, J. A. (2005). More frequent partner hugs and higher oxytocin levels are linked to lower blood pressure and heart rate in premenopausal women. Biol. Psychol. 69, 5-21.

Lupien, S. J., Fiocco, A., Wan, N., Maheu, F., Lord, C., Schramek, T., and Tu, M. T. (2005). Stress hormones and human memory function across the lifespan. Psychoneuroendocrinology 30, 225-242.

Pratt, J. J. (1978). Steroid immunoassay in clinical chemistry. Clin. Chem. 24, 1869-1890.

Rijsdijk, F. V., Riese, H., Tops, M., Snieder, H., Brouwer, W. H., Smid, H. G. O. M., and Ormel, J. (2009) Neuroticism, recall bias and attention bias for valenced probes: a twin study. Psychol. Med. 39, 45-54.

Rimmele, U., Hediger, K., Heinrichs, M., and Klaver, P. (2009). Oxytocin makes a face in memory familiar. $J$. Neurosci. 29, 38-42.

Risold, P. Y., and Swanson, L. W. (1996). Structural evidence for functional domains in the rat hippocampus. Science 272, 1484-1486.

Salonia, A., Nappi, R. E., Pontillo, M., Daverio, R., Smeraldi, A., Briganti, A., Fabbri, F., Zanni, G., Rigatti, P., and Montorsi, F. (2005). Menstrual cycle-related changes in plasma oxytocin are relevant to normal sexual function in healthy women. Horm. Behav. 47, 164-169.
Silber, M., Almkvist, O., Larsson, B., and Uvnäs-Moberg, K. (1990). Temporary peripartal impairment in memory and attention and its possible relation to oxytocin concentration. Life Sci. 47, 57-65.

Skrundz, M., Bolten, M., Nast, I., Hellhammer, D. H., and Meinlschmidt G. (2011). Plasma oxytocin concentration during pregnancy is associated with development of postpartum depression. Neuropsychopharmacology 36, 1886-1893.

Stock, S., and Uvnäs-Moberg, K. (1985). Oxytocin infusions increase plasma levels of insulin and VIP but not of gastrin in conscious dogs. Acto Physiol. Scand. 125, 205-210.

Stock, S., and Uvnäs-Moberg, K. (1988) Increased plasma levels of oxytocin in response to afferent electrical stimulation in sciatic and vagal nerves and in response to touch and pinch in anaesthetized rats. Acta Physiol. Scand. 132, 29-34.

Tops, M., Boksem, M. A. S., Wijers, A. A., van Duinen, H., Den Boer, J. A., Meijman, T. F., and Korf, J. (2007a). The psychobiology of burnout: are there two different syndromes? $\mathrm{Neu}$ ropsychobiology 55, 143-150.

Tops, M., van Peer, J. M., and Korf, J. (2007b). Individual differences in emotional expressivity predict oxytocin responses to cortisol administration: relevance to breast cancer? Biol. Psychol. 75, 119-123.

Tops, M., van Peer, J. M., Wijers, A. A., Korf, J., and Tucker, D. M. (2007c). Anxiety, cortisol and attachment predict plasma oxytocin. Psychophysiology 44, 444-449.

Tops, M., van der Pompe, G., Baas, D., Mulder, L. J. M., den Boer, J. A., Meijman, T. F., and Korf, J. (2003). Acute cortisol effects on immediate free recall and recognition of nouns depend on stimulus valence. Psychophysiology 40, 167-173.

Tops, M., van der Pompe, G., Wijers, A A., den Boer, J. A., Meijman, T. F., and Korf, J. (2004). Free recall of pleasant words from recency positions is especially sensitive to acute administration of cortisol. Psychoneuroendocrinology 29, 327-338.

Tops, M., van Peer, J. M., Wester, A. E., Wijers, A. A., and Korf, J. (2006). State-dependent regulation of cortical activity by cortisol: an EEG study. Neurosci. Lett. 404, 39-43.

Uvnäs-Moberg, K. (1998). Oxytocin may mediate the benefits of positive social interaction and emotions. Psychoneuroendocrinology 23, 819-835.

Wolf, O. T. (2009). Stress and memory in humans: twelve years of progress? Brain Res. 1293, 142-154.

Young, L. J. (2002). The neurobiology of social recognition, approach, and avoidance. Biol. Psychiatry 51, $18-26$.

Zola-Morgan, S. M., and Squire, L. R. (1990). The primate hippocampal formation: evidence for a timelimited role in memory storage. Science 250, 288-290.

Conflict of Interest Statement: The research was conducted in the absence of any commercial or financial relationships that could be construed as a potential conflict of interest.

Received: 21 December 2011; paperpending published: 27 January 2012; accepted: 20 April 2012; published online: 16 May 2012.

Citation: Tops M, Buisman-Pijlman FTA, Boksem MAS, Wijers AA and Korf $J$ (2012) Cortisol-induced increases of plasma oxytocin levels predict decreased immediate free recall of unpleasant words. Front. Psychiatry 3:43. doi: 10.3389/fpsyt.2012.00043

This article was submitted to Frontiers in Psychopharmacology, a specialty of Frontiers in Psychiatry.

Copyright (c) 2012 Tops, BuismanPijlman, Boksem, Wijers and Korf. This is an open-access article distributed under the terms of the Creative Commons Attribution Non Commercial License, which permits non-commercial use, distribution, and reproduction in other forums, provided the original authors and source are credited. 